\title{
Erratum: “Ferromagnetic order in dipolar systems with anisotropy: application to magnetic nanoparticle supracrystals" [Condens. Matter Phys., 2017, 20, No 3, 33703: 1-10, doi:10.5488/CMP.20.33703]
}

\author{
V. Russier, E. Ngo \\ ICMPE, UMR 7182 CNRS and UPE 2-8 rue Henri Dunant 94320 Thiais, France \\ Received August 3, 2018
}

The sentence bracketing equation (2.3), together with the first equality in equation (2.3) must be changed according to:

We also introduce more relevant coupling parameters through a reference distance, $d_{\text {ref }}=a \phi^{-1 / 3}$, and a reduced temperature

$$
\lambda_{d}=\epsilon_{d}\left(\frac{d}{d_{\mathrm{ref}}}\right)^{3}, \quad \lambda_{u}=\epsilon_{u} / \lambda_{d}, \quad T^{*}=T /\left(T_{0} \lambda_{d}\right),
$$

where $\phi$ is the volume fraction and a convenient choice for the proportionality constant is $a=\left(\phi_{M}^{(0)}\right)^{1 / 3}, \phi_{M}^{(0)}$ being the maximum value of $\phi$ for a given structure here taken as the fcc lattice $\left[\phi_{M}^{(0)}=\phi_{M}^{(\mathrm{fcc})}=\sqrt{2} \pi / 6\right]$.

\author{
Erratum: “Феромагнітний порядок у дипольних системах 3 \\ анізотропією: застосування до магнітних наночастинкових \\ супракристалів" [Condens. Matter Phys., 2017, 20, № 3, \\ 33703: 1-10, doi:10.5488/CMP.20.33703] \\ B. Рус'є, E. Нro \\ Інститут хімії та матеріалів при університеті “Париж-Схід” (УПС), дослідницький центр 7182 \\ Національного центру наукових досліджень та УПС, вул. Анрі Дюнана, 2-8, 94320 Тьє, Франція
}




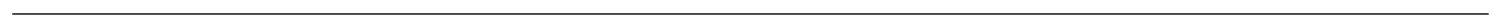

